Attosecond electron sheets and attosecond light pulses from relativistic laser wakefields in underdense plasmas

F. Y. Li, Z. M. Sheng' , M. Chen' , L. L. Yu, J. Meyer-ter-Vehn, W. B. Mori, and J. Zhang

Citation: 1777, 040015 (2016); doi: 10.1063/1.4965617

View online: http://dx.doi.org/10.1063/1.4965617

View Table of Contents: http://aip.scitation.org/toc/apc/1777/1

Published by the American Institute of Physics 


\title{
Attosecond Electron Sheets and Attosecond Light Pulses from Relativistic Laser Wakefields in Underdense Plasmas
}

\author{
F. Y. Li ${ }^{1}$, Z. M. Sheng, ${ }^{1,2}$ a), M. Chen, b), L. L. Yu ${ }^{1}$, J. Meyer-ter-Vehn ${ }^{3}$, W. B. \\ Mori $^{4}$, J. Zhang ${ }^{1}$
}

${ }^{1}$ Key Laboratory for Laser Plasmas (Ministry of Education) and Department of Physics and Astronomy, Shanghai Jiao Tong University, Shanghai 200240, China

${ }^{2} S U P A$, Department of Physics, University of Strathclyde, Glasgow G4 0NG, UK

${ }^{3}$ Max-Planck-Institut fuer Quantenoptik, D-85748 Garching, Germany

${ }^{4}$ Department of Physics and Astronomy and Department of Electrical Engineering, University of California, Los Angeles, California 90095-1547, USA

a)zhengming.sheng@strath.ac.uk or zmsheng@sjtu.edu.cn

b) minchen@sjtu.edu.cn

\begin{abstract}
A method for producing dense attosecond electron sheets (tens nanometers thick corresponding to attosecond duration) and single intense attosecond light pulse is reported based on relativistic laser wakefields in underdense plasmas. By making use of gas targets this method allows for high repetition rates required for applications.
\end{abstract}

\section{INTRODUCTION}

Generation of attosecond bursts of coherent extreme-ultraviolet (XUV) and soft x-ray pulses is presently a major goal in relativistic laser plasma physics [1]. So far, attosecond science usually relies on high harmonics from gas target [2]. In laser plasma interaction short light pulse generation is normally accompanied with short electron beams. Harmonics from laser solid interaction make use of relativistic dynamics of surface electron layers, which can generate much brighter sources [3]. These harmonic sources usual show trains pulses in time domain and isolate single spikes can be obtained through spectral filtering. Single attosecond light pulse can also be produced from relativistic electron sheets extracted from thin foils. These sheets can be used for coherent backscattering or selfemit single attosecond pulses [4].

In this paper, we present a new path for producing intense attosecond source from relativistic laser wakefields in underdense plasmas. Usually laser wakefield acceleration produces femtosecond long electron beams [5], which have been used to generate incoherent femtosecond betatron radiations or Thomson/Compton sources [6]. Here, we show that this picture changes dramatically when an ultrathin overcritical dense electron sheet is injected into the wakefield. The injected sheet contracts in transverse direction due to radial wake fields, while boosted in energy by longitudinal wake field, and is found to emit a strong unipolar attosecond electromagnetic pulse during the transverse contraction. As we shall see, this coherent attosecond burst differs in many aspects from the betatron radiations normally obtained [6]. 


\section{INJECTION OF ATTOSECOND ELECTRON SHEETS}

We first show how to inject a dense electron sheet into a wakefield. Various controlled electron injection schemes have actually been proposed for the generation of stable, monoenergetic electron beams [5]. However, usually these femtosecond, pC-charged beams are still continuously injected. On the other hand, the density singularities that arise in nonlinear plasma waves are very thin [7], so that, if the breaking of the wave can be controlled to occur in a limited space and time, the injected density spike could be the source of dense attosecond bunches with monochromatic spectra. For this to happen, we consider to use an upramp density profile followed by a plateau. The key elements are that, along the ramp, the wake wave travels at a superluminal phase speed $v_{p}$ even for high nonlinearities. Thus the density wave crests can be stably compressed into high density spikes without premature injection. This free of injection finally terminates as the wake propagates through the ramp to the following plateau where the phase speed becomes subluminal suddenly. As a result, a major part of the tightly compressed density crest can be trapped as a whole at the density transition region due to a fast switch of $v_{p}$ from above to below $\mathrm{c}$.
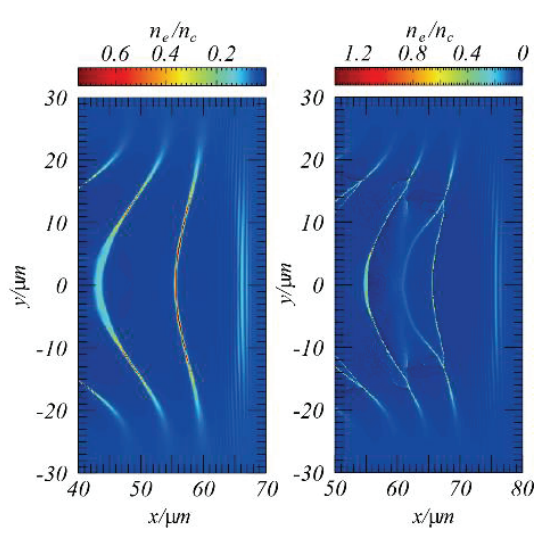

FIGURE 1. (a) Snapshots of electron density distribution when approaching or shortly after the ramp-plateau transition region, located at $\mathrm{x}=65 \mu \mathrm{m}$.

In high dimensions, a relatively broad focal spot of the driving laser (larger than the wake wavelength) is used to drive the wake in the quasi-one-dimensional (quasi-1D) regime, so that the above 1D dynamics can be preserved and a relativistic electron sheet is produced. This is different from the bubble regime occurring for tightly focused driving pulses, where the wake electrons are strongly evacuated and transverse injection at the rear vertex dominates, forming narrow accelerating bunches [8]. Details of the electron sheet injection are documented in Ref. [9]. Here, a sample 2D particle-in-cell (PIC) simulation [10] is given. A linearly polarized (along z axis), 18 fs laser pulse of peak intensity $\mathrm{I}_{0}=7.7 \times 10^{19} \mathrm{~W} / \mathrm{cm}^{2}$ irradiates an underdense plasma with a $65 \mu \mathrm{m}$ long ramping front. The plateau density is $\mathrm{n}_{0}=0.04 \mathrm{n}_{\mathrm{c}}$ with $\mathrm{n}_{\mathrm{c}}=1.74 \times 10^{21} \mathrm{~cm}^{-3}$ the critical density for laser wavelength $\lambda_{0}=0.8 \mu \mathrm{m}$. A laser waist of $\mathrm{W}_{0}=17 \mu \mathrm{m}$ is sufficient for the formation of electron sheets in the quasi-1D regime. An electron temperature of $\mathrm{T}_{\mathrm{e}}=20 \mathrm{eV}$ is initialized to mimic field ionization by prepulse. A snapshot of electron density around the sharp injection instant is given Fig. 1. They illustrate well the converging of the density crests when approaching the plateau region and the trapping of a dense attosecond electron sheet after the density transition.

\section{GENERATION OF ATTOSECOND LIGHT PULSE}

Figure 2 shows the evolution of the injected sheet in the wakefield and the associated radiation flash. It is seen that the sheet contracts transversely to a small diameter in tens of laser periods after injection. It is in this short time interval that the attosecond pulse is emitted [11]. As found in Fig. 2(b), the pulse is first attached to the electron sheet, but then propagates along a cone, while the electrons move closer to the axis in a channel of almost constant diameter. The attosecond emission is actually a single annulus beam in 3D geometry. What plotted in Fig. 2(b) are similar to projections of the pulse in the present $x-y$ plane. 
We analyze the radiation process in general. The injected electrons are diverted by focusing wake fields toward the central axis according to $\mathrm{d} \beta_{\perp} / \mathrm{dt} \simeq \epsilon_{\perp} / \gamma$. Here, $\beta_{\perp}$ is the normalized transverse velocity; $\mathrm{t}$ and the focusing field $\epsilon_{\perp}$ are respectively normalized by $\omega_{0}{ }^{-1}$ and $E_{0}=m_{e} \omega_{0} c / e$ with electron mass $m_{e}$, elementary charge e and laser frequency $\omega_{0}=2 \pi \mathrm{c} / \lambda_{0}$. Near the center axis, the focusing field, $\epsilon_{\perp} \propto \mathrm{n}_{0} \mathrm{r}$, is almost linear in both radius $\mathrm{r}$ and ambient density $\mathrm{n}_{0}$. Trapped electrons thus perform synchrotron-like motion with curvature radius $\rho \simeq \lambda_{0} \gamma / 2 \pi \epsilon_{\perp}$ and emit broad band radiation with cutoff frequency $\omega_{\mathrm{c}} \simeq 3 \gamma^{3} \mathrm{c} \rho^{-1}$ [12]. Meanwhile, there are a couple of new features when compared with the betatron radiations. First, the high density of the sheet, typically greater than $10^{21} \mathrm{~cm}^{-3}$, enables synchrotron radiation in a coherent manner. Coherence occurs provided that a sufficiently large number of electrons resides in a volume with a scale length equal to the radiation wavelength in the rest frame of electrons. For the present case, it requires the sheet density to satisfy $n_{s} \gg 10^{13} \epsilon_{\perp}^{3} \gamma^{4} \mathrm{~cm}^{-3}$, where radiation at the cutoff wavelength $\lambda_{\mathrm{c}}=2 \pi \mathrm{c} / \omega_{\mathrm{c}}$ is assumed. Considering $\epsilon_{\perp} \sim 0.1$, this criterion can be readily met even for $\gamma>100$. Second, due to the axial symmetry and the inward acceleration of disk electrons, radially polarized half-cycle electromagnetic pulses are produced. Third, with the increase of $\gamma$ by wakefield acceleration, the radiated power grows $\propto \gamma^{2}$. That means the major emission profile is roughly overlapped with the spatial distribution of sheet electrons during the amplification, and thereby naturally has an attosecond duration. It is interesting to notice that radially polarized half-cycle emissions have recently been obtained from thin photoconductors [13], where a sequence of annulus microelectrodes is fabricated to induce the radial currents. However, due to the static wafer plane, only pulses of picosecond duration are generated, determined by the lifetime of the radiating currents.

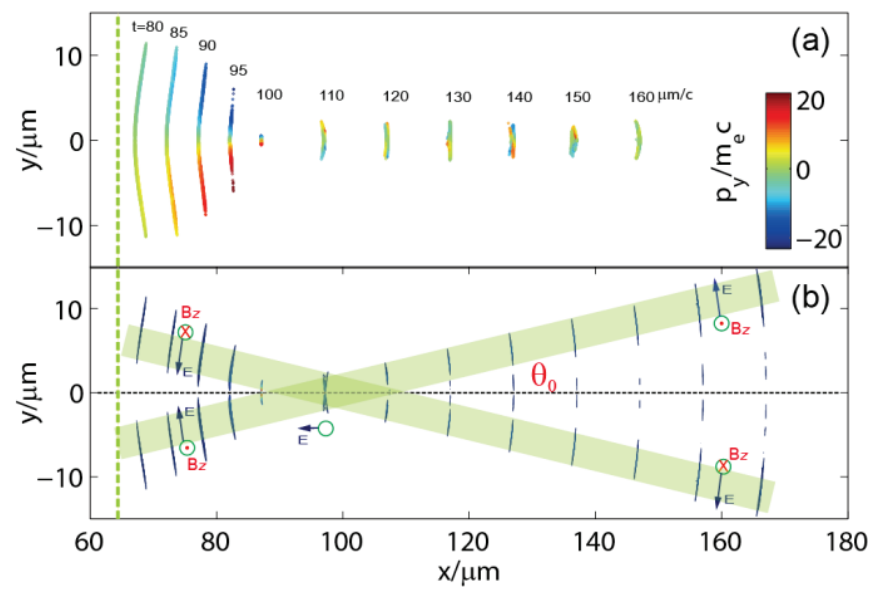

FIGURE 2. (a) Snapshots of trapped electron sheet at different times (colored according to transverse momentum $\mathrm{p}_{\mathrm{y}} / \mathrm{m}_{\mathrm{e}} \mathrm{c}$ ) and (b) the radiated attosecond flash given as contour plot of $\mathrm{B}_{\mathrm{z}}{ }^{2}$. The dashed line on the left side marks the position of sharp injection. In full 3D geometry, the emission appears as an annular beam propagating along a cone with opening angle $\theta^{0} \sim 10^{\circ}$, indicated as shaded stripes.

These features are well illustrated by the sample simulation. Figure 3(a) shows how the peak field of the radially polarized, half-cycle, attosecond (RHA) pulse evolves. According to Fig. 1(b), the attosecond pulse emerges in three stages: radiation amplification during initial contraction, beam crossing on axis, and finally annular beam propagation along a cone. At the phase of beam crossing, the emissions focus to a maximum of $\mathrm{B}_{\mathrm{z}}{ }^{\max } / \mathrm{B}_{0} \geq 18$ $\left(B_{0}=m_{e} \omega_{0} / e\right.$ is the normalizing field), corresponding to a peak intensity greater than $7 \times 10^{20} \mathrm{~W} / \mathrm{cm}^{2}$, almost 10 times larger than the driving pulse. After beam crossing on axis, e.g., $\mathrm{t}>130 \mu \mathrm{m} / \mathrm{c}$, the actual peak field shows up as $\mathrm{B}_{\mathrm{z}}{ }^{\max } / \mathrm{B}_{0}=4.5$, corresponding to $4.3 \times 10^{19} \mathrm{~W} / \mathrm{cm}^{2}$. It then slowly decays due to diffraction.

Figure 3(b) further shows how the RHA pulse propagates at later time. Due to laser evolution, the initial quasi1D wake is evolving into a complex broken-wave pattern [14]. The half-cycle pulse as short as 120 as [see Fig. 3(c)] propagates inside the wake, but deviated from the center axis. The corresponding spatial spectrum given in Fig. 3(d) shows clearly two branches, each with a small divergence and a broad-band XUV spectrum extending up to $\mathrm{k} / \mathrm{k}_{0} \simeq$ 25 , where $\mathrm{k}_{0}=2 \pi / \lambda_{0}$. In this sample case, the annular beam carries a total energy of $\sim 12 \mathrm{~mJ}$, which is $\sim 2 \times 10^{-3}$ of the incident laser energy. 
The RHA generation is insensitive to finite thermal temperatures. The emission profile hardly changes when increasing $\mathrm{T}_{\mathrm{e}}$ up to $100 \mathrm{eV}$ as depicted in Fig. 3(c). Also shown in this subfigure is the resulting emission profile when switching the polarization direction of the driving pulse from $\mathrm{z}$ to $\mathrm{y}$ (within the simulation plane) axis. Few differences are observed, indicating that the polarity of the attosecond emissions is independent of the driving pulse, but only determined by the transverse contraction motion of the electron sheet. We have confirmed using 3D PIC simulations (not shown here) that the annular attosecond pulse is uniform in intensity azimuthally, corresponding to radial polarization.
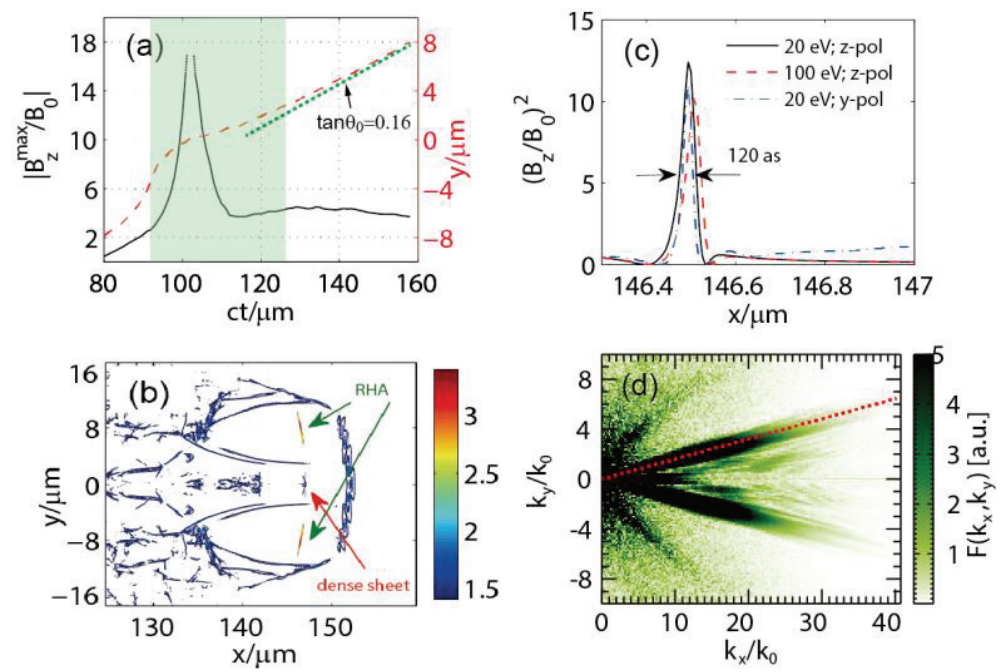

FIGURE 3. (a) Evolution of peak field $\mathrm{B}_{\mathrm{z}}{ }^{\mathrm{max}}$ (solid) and associated y coordinate (dashed) of the RHA pulse. The shaded region refers to the beam crossing stage as explained in the text. The green dotted line indicates the slope of propagation direction. (b) Contour plots of the electron density $n / n_{c}$ and $\left|B_{z} / B_{0}\right|$ (share the same colorbar) at $t=$ $160 \mu \mathrm{m} /$ c. (c) Corresponding lineouts of $\left(B_{z} / B_{0}\right)^{2}$ along $y=8.32 \mu \mathrm{m}$ for three cases corresponding to different initial plasma temperatures $(20$ and $100 \mathrm{eV})$ and y or $\mathrm{z}$ polarization of driving laser pulse. (d) Corresponding 2D spatial spectrum of the RHA pulse.

\section{SIMPLIFIED MODEL FOR RADIATION GENERATION}

The basic dynamics of RHA generation can be derived using a simplified model. It assumes that a flat, monoenergetic electron sheet of delta-like density profile $\mathrm{n}_{\mathrm{s}}=\delta\left(\mathrm{x} / \sigma_{\mathrm{s}}\right)$ with finite areal density $\sigma_{\mathrm{s}}=\int \mathrm{n}_{\mathrm{s}} \mathrm{dx}$ and no initial transverse momentum is injected into a wakefield of uniform accelerating and focusing fields. Coherent radiation from the electron sheet is then described by the $1 \mathrm{D}$ wave equation $\left(\partial^{2} / \partial \mathrm{x}^{2}-\mathrm{c}^{-2} \partial^{2} / \partial \mathrm{t}^{2}\right) \mathrm{E}_{\mathrm{y}, \mathrm{r}}=$ $\epsilon_{0}{ }^{-1} c^{-2} \partial J_{y, r} / \partial t$, where $J_{y, r}=-e c \beta_{y, r} n_{s}$ is the radiating current with $\beta_{y, r}=\int_{\tau_{0}}^{\tau_{1}} d \beta_{y}$ the velocity integrated over a short transverse acceleration. The radiated field $\mathrm{E}_{\mathrm{y}, \mathrm{r}}$ can be first calculated in the rest frame of the sheet with Lorentz factor $\sim \gamma$ and then transformed back to the laboratory frame. The result is given by $\mathrm{E}_{\mathrm{y}, \mathrm{r}} \simeq\left(\sigma_{\mathrm{s}} / \epsilon_{0}\right) \beta_{\mathrm{y}, \mathrm{r}}{ }^{\mathrm{R}} \gamma$, where $\beta_{y, r}{ }^{R}$ denotes the velocity $\beta_{y, r}$ in the rest frame. This indicates that the radiated power grows rapidly with the electron energy, which follows from $d \gamma / d t=-\beta_{x} \cdot E_{x}-\beta_{y} \cdot E_{y}$. Taking into account both the radiated fields and the wake fields, the energy equation can be rewritten as $d \gamma / d t+\left(\sigma_{s} / \epsilon_{0}\right) \beta_{y, r}{ }^{R} \beta_{y} \gamma-\beta_{y} \cdot \mathrm{E}_{\mathrm{y}, \mathrm{w}}-\beta_{\mathrm{x}} \cdot \mathrm{E}_{\mathrm{x}, \mathrm{w}}=0$, where $E_{x, w}$ and $E_{x, w}$ are the longitudinal and transverse wake fields, respectively. Assuming $\left.\gamma\right|_{t=0}=1$ initially, wakefield acceleration first boosts $\gamma$-factor rapidly, which then saturates due to radiation damping at a rate $\left(\sigma_{\mathrm{s}} / \epsilon_{0}\right) \beta_{\mathrm{y}, \mathrm{r}}^{\mathrm{R}} \beta_{\mathrm{y}}$. Saturation occurs for $\beta_{\mathrm{x}} \cdot \mathrm{E}_{\mathrm{x}}+\beta_{\mathrm{y}} \cdot \mathrm{E}_{\mathrm{y}}=0$, which leads to the saturated field amplitude $\mathrm{E}_{\mathrm{y}, \mathrm{r}}{ }^{\text {sat }} \simeq$ $\mathrm{E}_{\mathrm{x}, \mathrm{w}} / \tan \theta+\mathrm{E}_{\mathrm{y}, \mathrm{w}}$. Here, $\tan \theta=\beta_{\mathrm{y}} / \beta_{\mathrm{x}}$ is evaluated at the saturation instant. It is clear that wakefield acceleration has played an essential role in RHA generation. 


\section{SUMMARY}

In conclusion, we have reported a new method for producing attosecond light pulse from attosecond electron sheet formed in relativistic laser wakefields. The attosecond feature does not derive from solid surfaces [3] or thin foils [4], but arises from intrinsic features of nonlinear plasma waves, namely steepening and breaking. It makes use of quasi-1D wavebreaking, allowing to trap an ultrathin electron sheet into the wakefield using an up-ramp density transition. The electron sheet contracts in transverse direction, while boosted in energy, and is found to emit an intense half-cycle attosecond pulse. Pulse energy exceeding $10 \mathrm{~mJ}$ can be obtained with the laser-to-RHA conversion efficiency exceeding $10^{-3}$. Besides being a bright source for attosecond applications, this burst will also provide useful diagnostics for wavebreaking dynamics [15] in the highly nonlinear quasi-1D regime.

\section{ACKNOWLEDGMENTS}

Z.M.S. thanks the OSIRIS Consortium at UCLA and IST for providing access to OSIRIS 2.0 framework. F.Y.L. acknowledges fruitful discussions with Prof. Hui-Chun Wu. M.C. thanks supports from National 1000 Youth Talent Project of China and supports by Shanghai Science and Technology Commission (Grant No. 13PJ1403600). This work was supported in part by the National Basic Research Program of China (Grant No. 2013CBA01504), the National Natural Science Foundation of China (Grant No. 11121504, 11374209 and 11374210), and the MOST international collaboration project (Grant No. 2014DFG02330). Simulations were supported by the Shanghai Supercomputer Center and the center for high performance computing at SJTU.

\section{REFERENCES}

1. F. Krausz and M. Ivanov, Rev. Mod. Phys. 81, 163 (2009).

2. P. B. Corkum and F. Krausz, Nat. Phys. 3, 381 (2007).

3. U. Teubner and P. Gibbon, Rev. Mod. Phys. 81, 445 (2009).

4. J. Meyer-ter Vehn and H.-C. Wu, The European Physical Journal D-Atomic, Molecular, Optical and Plasma Physics 55, 433 (2009); D. Kiefer, M. Yeung, T. Dzelzainis, P. Foster, S. Rykovanov, C. L. Lewis, R. Marjoribanks, H. Ruhl, D. Habs, J. Schreiber, et al., Nature communications 4, 1763 (2013); H. C. Wu and J. Meyer-ter-Vehn, Nat. Photon. 6, 304 (2012).

5. E. Esarey, C. Schroeder, and W. Leemans, Rev. Mod. Phys. 81, 1229 (2009).

6. S. Corde, K. Ta Phuoc, G. Lambert, R. Fitour, V. Malka, and A. Rousse, Rev. Mod. Phys. 85, 1 (2013).

7. A. Akhiezer and R. Polovin, Sov. Phys. JETP 3, 696 (1956).

8. A. Pukhov and J. Meyer-ter-Vehn, Appl. Phys. B 74, 355 (2002); W. Lu, C. Huang, M. Zhou, W. B. Mori and T. Katsouleas, Phys. Rev. Lett. 96, 165002 (2006).

9. F. Y. Li, Z. M. Sheng, Y. Liu, J. Meyer-ter-Vehn, W. B. Mori, W. Lu and J. Zhang, Phys. Rev. Lett. 110, 135002 (2013).

10. R. Fonseca et al., Lecture Notes in Computer Science. 2331, 342-351 (Springer Berlin, Heidelberg, 2002).

11. F. Y. Li, Z. M. Sheng, M. Chen, L. L. Yu, J. Meyer-ter-Vehn, W. B. Mori, and J. Zhang, arXiv:1401.2799.

12. T. Shiozawa, Classical Relativistic Electrodynamics, Springer-Verlag (Berlin, Heidelberg, 2004), pp77-82.

13. K. Kan, J. Yang, A. Ogata, S. Sakakihara, T. Kondoh, K. Norizawa, I. Nozawa, T. Toigawa, Y. Yoshida, H. Kitahara, K. Takano, M. Hangyo, R. Kuroda and H. Toyokawa, App. Phys. Lett. 102, 221118 (2013).

14. J. Xu, B. Shen, X. Zhang, M. Wen, L. Ji, W. Wang, Y. Yu and K. Nakajima, New J. Phys. 12, 023037 (2010).

15. A. G. R. Thomas, S. P. D. Mangles, Z. Najmudin, M. C. Kaluza, C. D. Murphy, and K. Krushelnick, Phys. Rev. Lett. 98, 054802 (2007). 